\title{
Assessment of allergen-specific IgE by immunoblotting method in atopic dermatitis
}

\author{
${ }^{1}$ Immunology Research Center, Faculty of medicine, Tabriz University of Medical Sciences, Tabriz, Iran \\ ${ }^{2}$ Umbilical Cord stem cell research Center, Tabriz University of Medical Sciences, Tabriz, Iran \\ ${ }^{3}$ Cellular and Molecular Research Center, Yasuj University of Medical Sciences, Yasuj, Iran
}

\section{KEY WORDS}

Atopic dermatitis; allergen; IgE;

Immunoblotting; Immunotherapy

\section{Corresponding author}

Nayer Seyfizadeh

Umbilical Cord Stem Cell Research Center

Tabriz University of Medical Sciences

Tabriz, Iran

E-mail: Seyfizadehn@tbzmed.ac.ir

Doi

10.23822/EurAnnACI.1764-1489.06

\begin{abstract}
Summary
Background and Objectives. Stimulating the immune system by exposure to various allergens to produce specific IgE has a significant role in the pathogenesis of atopic dermatitis. Identifying disease-causing allergens, prevention of exposure to those allergens, and immunotherapy will play an important role in the treatment of Atopic Disease. The purpose of this study was to determine the common allergens of northwest of Iran in patients with atopic dermatitis that are resistant to treatment. Materials and methods. In this descriptive-analytical study, serum levels of total IgE and frequency of specific IgE were measured by Immunoblotting against 20 common allergens in 150 cases of patients with atopic dermatitis, attending to dermatology and asthma and allergy clinics from 2010 to 2011. The control group consisted of individuals who had been clinically healthy. Results. In the 90\% of patients that were included in this study, total IgE levels were higher than in healthy people with mean serum levels of total IgE $227.51 \pm 103 \mathrm{IU} / \mathrm{ml} .136$ patients (90.6\%) had specific IgE for at least one allergen. The frequency of positive allergens among the patients who were included in this study were $53.34 \%, 26.8 \%$, and $19.56 \%$ respectively in plants and fungus allergens group, animal allergens group and food allergens group. After avoiding of the allergens (which they had been sensitized to), 60\% of patients were cured with immune therapy, and total IgE serum levels in the control group were not increased. Conclusion. Identifying the abundant allergens such as cultivated rye, Timothy grass, house dust mite, birch, cat, horse, potato, dog, egg white, cow milk, in order to advise patients to avoid them or to do immunotherapy and desensitization, is useful in this area.
\end{abstract}

\section{Introduction}

Atopic dermatitis as a chronic inflammatory disease is characterized by papulovesicular and eczematous eruptions, which are recurrent and itchy. Because of severe eczema and nerve crushing itch, most of these patients are disabled in doing chores, typically have difficulties in their social relationships, and might also face some familial and mental problems as well.

This disease involves face, trunk and extensor surfaces in neonates and infants. However, the flexor surfaces of the extremities are in- volved in adults and juveniles (1). It is associated with activation of different immunological and inflammatory pathways, and it leads to increasing of T lymphocytes, Langerhans cells (APC) stimulation, and overproduction of specific IgE by B cells. Exposure to different allergens, and subsequently stimulation of Langerhans cells and $\mathrm{Ag}$ presentation by them to Th2 cells, causes the release of IL4, IL5, IL9, IL10, IL13, which results in B cell proliferation and consequent increasing of the production of specific IgE. Specific $\mathrm{IgE}$ plays an important role in the allergic reactions of people who 
are sensitive to the allergens, and can also exacerbate symptoms and stimulate the immune system more and more (2).

The overall prevalence rate is estimated about $10-15.6 \%$, and it is about $20 \%$ for children (3). In an investigation that has been done in the United States, the prevalence of $\mathrm{AD}$ for school-age children has been estimated about $17 \%$ (4). In another investigation, the prevalence of atopic dermatitis in years before 1960 was reported about 2-3\%; however, this parameter in 1960-70 has been increased up to $9-12 \%$ (5).

Half of the children with $\mathrm{AD}$ before age 7 have shown asthma or rhinitis (2). About $80 \%$ of the children with $\mathrm{AD}$ have shown inflammatory diseases, such as allergic rhinitis and asthma (6). Allergens have a major role in stimulating the immune system and causing the disease (7).

There are newer methods to measure the serum levels of specific $\mathrm{IgE}$. It is typically measured by some methods such as Radioallergosorbent test (RAST) and Enzyme immunoassay test (EIA) (7). Specific IgE antibodies show diagnostic values against various allergens, and previous studies reported that the correlation of specific IgE level with the disease severity is more significant than the total IgE level (5).

Warner et al (1997) conducted a study on 817 children showing atopic dermatitis diagnose. They showed a direct relationship between increased total $\operatorname{IgE}$ level and the severity of the disease, by measuring total $\operatorname{IgE}$ and specific $\operatorname{IgE}(8)$.

In 2004, Perry et al studied the relationship between the specific IgE levels using the CAP_RAST and oral food challenge tests. Based on the test results on 391 children, they concluded that the measurement of specific IgE in milk, egg, peanut, wheat, soybean and use of oral food challenge tests is considered an effective method to determine the causes of the allergic disease and AD determination (9).

More recently, Hon et al in a study on 117 children below 18 years investigated to find whether any age-specific IgE levels in children with $\mathrm{AD}$ is associated with more severe symptoms or not. They have shown that high levels of age-specific IgE are associated with the development and severity of AD (10).

Almost in all of the previous works, the specific antibodies have been measured by some old methods and only in certain allergens in patients with $\mathrm{AD}$. In this study, we measured the serum level of specific IgE for several allergens by immunoblotting, in order to identify the primary allergens that cause $\mathrm{AD}$ in the patients. We aim to measure the specific $\operatorname{IgE}$ level of twenty allergens in patients with atopic dermatitis who are resistant to the treatment by an immunoblotting method, in order to identify the prevalent allergens which are responsible for the disease.

\section{Materials and methods}

In this descriptive-analytical study, we aimed to determine total and specific serum level of IgE caused by twenty most fre- quent allergens in $150 \mathrm{AD}$ patients diagnosed, by using the Hanifin-Rajka criteria in order to evaluate the importance and frequency of that allergen. 150 healthy people subject to total IgE measurement, and 92 healthy individuals for specific IgE, were selected as the control groups. The study was approved by the ethical committee of Tabriz University of Medical Sciences.

\section{Sampling}

According to the standard principles, informed consent was obtained from all AD patients. Pregnant and breastfeeding women, and people with other diseases, were excluded from the study. Control groups included people who did not have AD and had been diagnosed healthy by a dermatologist. Samples were selected from atopic patients that referred to the dermatology clinics and asthma clinics in East Azerbaijan of Iran. The standardized questionnaire which includes the demographic information, personal or family history, disease duration, clinical symptoms, was filled by the patients. Serum samples from all patients prior to the treatment were taken in order to measure total $\operatorname{IgE}$ and specific IgE. Before analyzing total and specific $\mathrm{IgE}$ in serum, control and patient samples were stored at $-80^{\circ} \mathrm{C}$.

\section{ELISA test}

Total and specific IgE measured according to the manufacturer's instructions of kit (Monobind Inc, Lake Forest, USA). We measured serum levels with the predefined standard steps in the manual (i.e., 0, 10, 50, 100, 200, and 500). According to this investigation, we acquired the normal serum range, about 0 to $46 \mathrm{IU} /$ $\mathrm{mL}$, and 0 to $75 \mathrm{IU} / \mathrm{mL}$, for infants $(<2)$ and adults respectively.

\section{Immunoblotting test}

We selected 20 allergens, including food allergens (egg whites, milk, fish, flowers, wheat, rice, soybeans, nuts, carrots, potatoes and apples), plant and fungal allergens (Timothy grass, cultivated rye, birch, sagebrush, Cladosporium, and Alternaria), animals (cats, horses, dogs, house dust mite). For the determination of specific IgE against allergens, the German business kit (Euroimmun) was used. In this method, patients' serum is tested directly and based on the manufacturer's instructions in the manual. Strips of blotting after staining are scanned and then analyzed with the Euroimmun's special software, figure 1.

At the same time, other discussed items, including some demographic information (age, gender, and occupation), primary and secondary standards and clinical symptoms of atopic dermatitis, were considered and evaluated. We used a descriptive statistical method to investigate the mean values, their standard deviations and evaluate the correlation among different factors that we have mentioned earlier. The analysis has been conducted by using the 
Figure 1-Immunoblotting strip of Atopic patients.

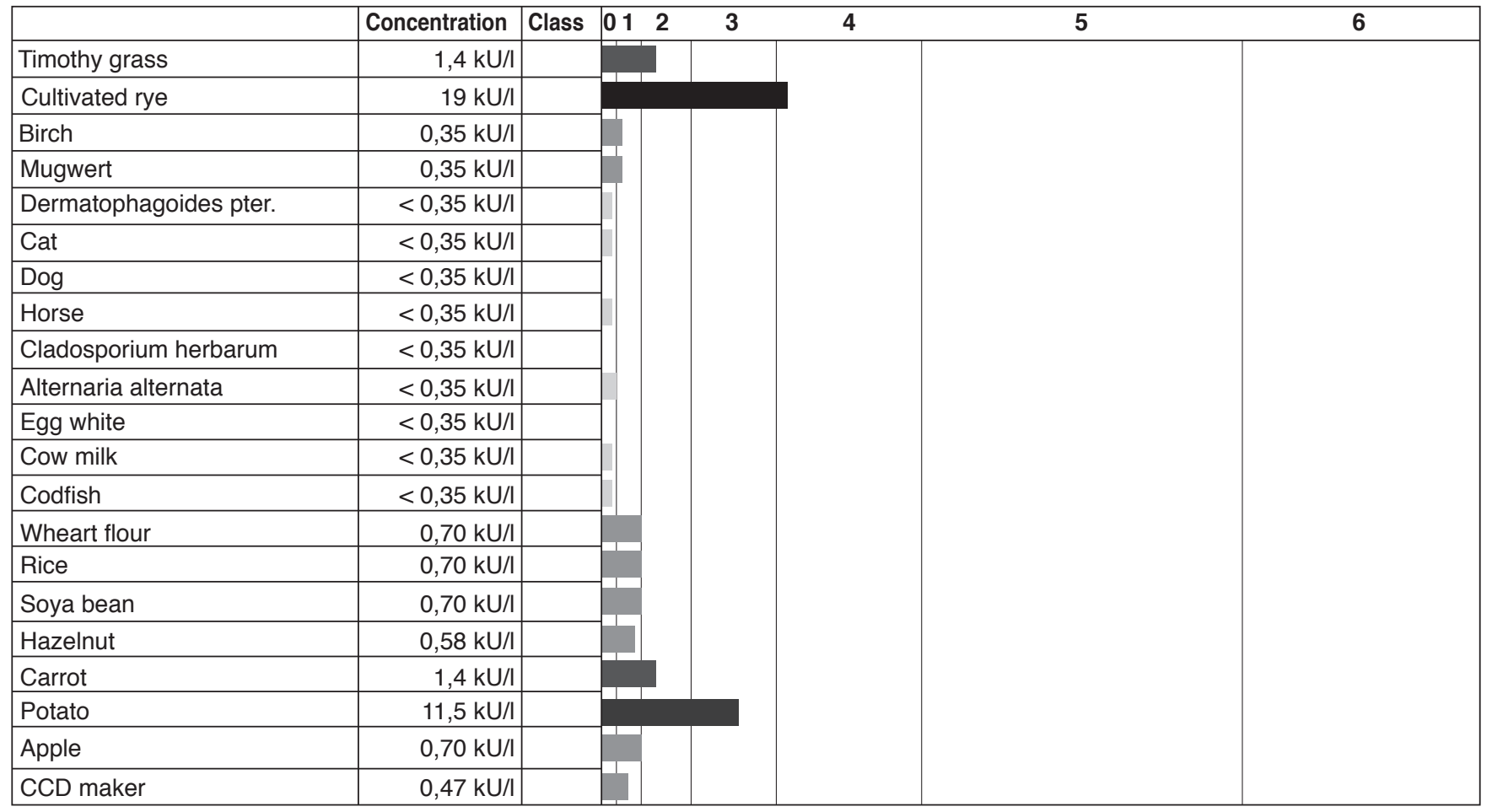

Statistical Product and Service Solutions (SPSS) software version 13.0, which is a powerful numerical tool for statistical studies. The standard chi-square test and Fisher's exact test are applied to the obtained data, to estimate the probability of exceeding.

\section{Results}

The mean age of 150 selected patients was $30.2 \pm 14.7$ years. Patients were classified into three sub-groups: $0-2$ years old, i.e. infants $(8.6 \%), 2-12$ years old, i.e. children $(11.4 \%)$, and 12 years and older, i.e. adults (80.0\%). $51.3 \%$ of patients were male and $48.7 \%$ of patients were female. This study indicated that $100 \%$ of the youngest sub-group (i.e. infants) including itching considered as the most important factor in the Hanifin-Rajka criteria. The involved areas were face, neck, and the extensor areas, but flexor areas were not involved in any of the infants. Children and adult patients showed itching symptoms in these areas, as follows: $90.0 \%$ in flexor areas, $9.3 \%$ in the face, $3.1 \%$ in the neck, and $18.5 \%$ in the extensor areas.

History of patients showed that 24 cases (16.0\%) had experienced allergic diseases (asthma, allergic rhinitis) and 11 atopic allergy patients, including allergic rhinitis disease too (7.3\%). The frequency of each sub-criterion is listed in table $\mathbf{1}$. Mean of serum levels of total IgE was $227.5 \pm 103.0$. The mean of total IgE levels in men and women was respectively $233.8 \pm 97.1$, and $221.6 \pm 111.5$. T-test results showed that there are no statistically significant differences between men and women in terms of total IgE levels $(p=0.74)$. Although, we must note the highly scattered serum levels around the mean values, and take into account this as we interpret these results. The mean value of the serum for the total $\operatorname{IgE}$ was $55.0 \pm 15.5$ in the control group. This suggests that $90 \%$ of the patients had total $\mathrm{IgE}$ levels higher than the mean serum value in the normal group. We report that $91 \%$ of the patients had specific $\operatorname{IgE}$ for at least one allergen. The patients, as mean to $3.06 \pm 2.31$ of the total of 20 tested allergens, had allergen-specific IgE. We found that the most abundant allergens related to $\mathrm{AD}$ are cultivated rye (48.6\%), Timothy grass (42.6\%), house dust mites (22.7\%) dog (16.7\%), birch $(11.3 \%)$, potato $(11.3 \%)$, horse $(10 \%)$, hazelnut $(10 \%)$, cat (10\%), soybean $(10 \%)$, sagebrush $(10 \%)$. In contrast, we found that apple, carrot, fish, wheat, rice, Cladosporium herbarum, cow milk, egg white, Alternaria had a low frequency (table 2).

The frequency of tested allergens by gender classification is presented in table 2 . Cat allergens were significantly more frequent in women $(p=0.02)$. Alternaria was significantly more common in men than women $(\mathrm{p}=0.042)$. The frequency of tested allergens in patients in plants and fungi group, animal group and food group was $54.34 \%, 26.08 \%$, and $19.56 \%$, respectively. 
Table 1 - Distribution of patients with minor symptoms of atopic dermatitis.

\begin{tabular}{lclc}
\hline Minor symptoms & Frequency $\%$ & Minor symptoms & Frequency $\%$ \\
\hline Familial allergy background & $24(16)$ & Itching in body & $150(100)$ \\
\hline Allergic rhinitis experiencing & $51(84)$ & Itching of the upper limbs & $75(50)$ \\
\hline $\begin{array}{l}\text { Associated Atopy with allergic } \\
\text { rhinitis }\end{array}$ & $11(7.3)$ & Itching of the upper limbs & $45(80)$ \\
\hline Disorders in digestive system & $6(4)$ & Itching in all over the body & $30(20)$ \\
\hline Hives & $6(4)$ & Throat itching & $6(4)$ \\
\hline Red rash & $99(66)$ & Ear itching & $11(7.3)$ \\
\hline Eczema & $135(90)$ & Eye itching & $9(6)$ \\
\hline Increased Total IgE & $135(90)$ & & \\
\hline Skin dryness & $81(54)$ & & \\
\hline
\end{tabular}

Table 2 - Distribution of sensitivity to any of the tested allergens in patients with atopic dermatitis.

\begin{tabular}{lccc}
\hline Allergens & Sum of patients (\%) & Women (\%) & Men (\%) \\
\hline Timothygrass & $64(42.6)$ & $34(46.5)$ & $30(38.9)$ \\
\hline Cultivated rye & $78(48.6)$ & $39(53.4)$ & $34(44.1)$ \\
\hline Birch & $17(11.33)$ & $5(6)$ & $12(5.5)$ \\
\hline Sagebrush & $15(10)$ & $7(9)$ & $8(10.4)$ \\
\hline $\begin{array}{l}\text { Dermatophagoides pteronyssinus } \\
\text { (house dust mite) }\end{array}$ & $34(22.7)$ & $26(35.6)$ & $4(5)$ \\
\hline Cat & $15(10)$ & $11(15)$ & $8(10.4)$ \\
\hline Dog & $25(16.7)$ & $17(23.2)$ & $8(10.4)$ \\
\hline Horse & $15(10)$ & $7(9)$ & $4(5)$ \\
\hline Cladosporium herbarum & $9(6)$ & $5(6)$ & $8(10.4)$ \\
\hline Alternaria alternate & $12(8)$ & $4(5)$ & $5(6.4)$ \\
\hline Egg white & $13(8.7)$ & $8(10.9)$ & $3(3.8)$ \\
\hline Cow milk & $13(8.7)$ & $10(13.6)$ & $0(0)$ \\
\hline Fish & $4(4.2)$ & $4(5)$ & $7(\%)$ \\
\hline Wheat & $13(8.7)$ & $6(\%)$ & $9(\%)$ \\
\hline Rice & $12(8)$ & $3(\%)$ & $6(7.7)$ \\
\hline Soybean & $15(10)$ & $9(12.3)$ & $8(10.4)$ \\
\hline Hazelnut & $15(10)$ & $7(9)$ & $6(7.7)$ \\
\hline Carrot & $11(7.3)$ & $5(6)$ & $6(7.7)$ \\
\hline Potato & $17(11.3)$ & $78(48.7)$ & $77(51.3)$ \\
\hline Apple & $11(7.3)$ & $5(6)$ & \\
\hline Total numbers & 150 & & \\
\hline
\end{tabular}




\section{Discussion}

We have assessed the common allergens of northwest of Iran in patients with atopic dermatitis that were resistant to treatment. Specific $\mathrm{IgE}$ antibodies have diagnostic values against various allergens, and a correlation between specific IgE level and disease severity was reported, and is more significant than total IgE level (8). The importance of identifying the presence of $\mathrm{IgE}$ antibody to any allergen in patients with atopic dermatitis, even in the absence of other clinical manifestations, could consist in allowing the prevention of accidental exposure and in educating in such prevention (11).

We have used Immunoblotting method in this study, while skin prick test is rapid and low cost, but has some limitations in children lower than 4 years old, and in the patients who are using antihistamines or suffering from serious atopic eczema. Specific IgE tests are more reliable and reproducible for the entire patients, without side effect risks, but 1 to 2 days are needed to reach to the result. ImmunoCAP Immuno-Solid Phase Allergy Chip could assess multiple allergen components on one slide, providing more detailed information than single IgE testing, but its clinical effectiveness and cost-effectiveness need to be more investigated $(12,13)$.

Our results show that atopic dermatitis can happen at any age, but it generally appears during childhood and infancy; it includes periods of exacerbations and improvement, but recovery will be achieved with increasing in age. These results are in agreement with the previous works $(1,7,14)$. Kulthanan et al conducted a similar study in Thailand with a focus on the clinical symptoms of patients with $\mathrm{AD}$. They reported an average age of the patients as $34.1 \pm 11.7$ years. According to the several previous studies the prevalence of atopic dermatitis is approximately considered $13-47 \%$ in adults $(15,16)$.

We used the age categorization suggested by Mgeladze et al to classify our patients and control groups, which were mostly adults (51.3\% male and $48.7 \%$ female) (17). In this study, primary and secondary criteria of itching were observed in all patients and $100 \%$ of cases were in consistent with the previous studies (18). According to various studies, compared to other methods the Hanifin-Rajka criteria for diagnosing of atopic dermatitis comprise the same high sensitivity and specificity of detection, and we also used these criteria in this study (19). Involved areas in atopic dermatitis in this study were: flexor in $90 \%$ of patients, face in $9.25 \%$ of patients, neck in $3.12 \%$ of patients and extensor areas in $8.5 \%$ of the patients. The study by Kulthanan et al in Thailand showed that characteristic morphology of disease was clear in $73.5 \%$ of patients. They reported the involved areas as the flexor areas, neck, extensor areas, and face in $72.1 \%, 27 \%, 18.5 \%$, and $18.1 \%$ of patients, respectively $(15,16)$. These percentages are slightly lower in comparison to the results that we presented in the previous section.

From another point of view, the difference in the percentage of involved areas in the adults by this work and Kulthanan et al might also be affected by the difference in the regions that the studies are conducted in, where people have different lifestyles. In the evaluation of minor criteria, we observed that the incidence of itching, skin dryness, itching of upper limbs, itching of lower extremities, hives, eczema, maculopapular rash, eye itching, were respectively $100 \%, 54 \%, 50 \%, 30 \%, 4 \%, 90 \%, 46 \%$, $6 \%$ among the patients. In many respects, there are similarities between different studies in the prevalence of minor criteria, and the differences can be attributed to the genetic and ethnic differences. In a study in Sweden by Bohme, due to the dry and cold weather, $100 \%$ of patients had dry skin (20). While a study by Wahab in Bangladesh, with warm and humid weather, showed that $43.8 \%$ of patients have dry skin (21). Kulthanan et al in Thailand studied clinical symptoms of 56 patients with atopic dermatitis and showed that patients have minor symptoms such as itching during sweating (67.3\%), skin dryness (67.3\%), nonspecific dermatitis of the hands and feet (34.7\%), frequent conjunctivitis $(20.2 \%)$, skin infection $(4.1 \%)$. Keratoconus was not seen in any cases $(15,16)$. The current study showed similar results as Kulthanan and colleagues.

135 (90\%) of cases in our patient group had higher serum levels of total IgE than normal, and average of total $\mathrm{IgE}$ serum levels, regardless of age and gender, was $227.5 \pm 103.0$. According to the previous studies, approximately $60-80 \%$ of patients have increased levels of total $\mathrm{IgE}$ than the general population $(1,3,10,15,20)$. Results from this study on the serum levels of total $\operatorname{IgE}$ are in agreement with the previous studies. Most frequent allergens were related to cultivated rye $(48.6 \%)$, Timothy grass $(42.6 \%)$, house dust mites $(22.7 \%)$, dog $(16.7 \%)$, birch $(11.3 \%)$, potato $(11.3 \%)$, horse $(10 \%)$, hazelnut $(10 \%)$, cat $(10 \%)$, soybean $(10 \%)$, sagebrush $(10 \%)$.

AhmadiAfshar et al (2008) investigated on the allergic patients with $\mathrm{AD}$. They reported the frequency of observed allergens in Zanjan-Iran as follows: grass pollen 38.5 to $41 \%$, weed pollen $21-27 \%$, olive trees $22 \%$, ash $20 \%$, house dust mite $16 \%$, cockroach $14.5 \%$, Aspergillus 11.5\%, Alternaria (7.5\%) (22).

Worm and colleagues (2005) conducted a study on 111 adult patients in Germany through the Prick test. They showed allergic reactions to birch $(55.6 \%)$, grass pollen $(44.4 \%)$, dog $(40.7 \%)$, potato $(37 \%)$, Cat $(33.3 \%)$, carrot $(29.6 \%)$, hazelnut (29.6\%), sesame (25.9\%), house dust mite $(22.2 \%)$, wheat flour $(22.2 \%)$, apple $(22.2 \%)$, parsley $(22.2 \%)$, sagebrush $(18.5 \%)$, barley flour (14.8\%), soybean (11.1\%), egg, cow milk and poppy seed each $7.4 \%$, peanut, pig meat, muscle meat, latex and Alternaria 3.7\%, Cladosporium, crab and a special type of fish have not been found in any of the patients (23).

Celakovska and colleagues in Paraguay studied 120 adult patients with AD using two methods: Atopy patch test and Evaluation of specific IgE with EIA method. Their investigations showed that the allergy frequency observed with both methods 
was as follows: wheat and soybeans, eggs, peanuts, cow's milk $4 \%, 8 \%, 13 \%, 1 \%$, respectively (24). Moreover, Hon and coworkers in a study of 85 children with atopic dermatitis (assessed by EIA) in China observed the food allergy occurrence in their patient population as fellow: shrimp 54\%, egg whites $43 \%$, wheat $42 \%$, peanuts $41 \%$, tomatoes $38 \%$, milk $34 \%$, fish $30 \%$, vea land orange $20 \%$ (25).

In summarizing the results of other studies, it seems that prevalence of food allergens in children is more than in adults, and about $35 \%$ or $1 / 3$ of children with severe atopic dermatitis have increased the level of $\operatorname{IgE}$ against food allergens. It is mostly believed that the lack of resistance evolution in children is the responsible factor which by growing to adulthood $80 \%$ of studied group did not show the food allergy. While the pollen and respiratory materials are more allergic in adults, the food allergens have less importance in them (23). In this study, $80 \%$ of investigated patients were adult and pollen and respiratory allergen (house dust mite, cat and fungi allergens) considered as an allergen and the food allergen could be considered as the less importance factor.

We emphasise the conclusion made by Worn investigation in Germany (23) and Celakovska et al in Paraguay (24) conducted on adults' allergies. They concluded that the frequency of plant and respiratory allergen in adults is higher than in the younger population. We found that the most frequent allergens are pollen of rye and Timothy grass. We conclude that the three most effective methods for the treatment of $\mathrm{AD}$ that we found are 1 - avoiding contact with the mentioned factors in pollen seasons (i.e. spring and early summer), 2 - increasing the drug dose in treatment, and particularly, 3 - the use of immunotherapy.

We found food allergy to potato is the most frequent observed allergen in food allergens group in the adult group, and that is consistent with Worn et al conclusion on the German patients. There are two possible explanations for that. The first idea is that the rate of potato consumption is high in both countries (i.e. Iran and Germany). In fact, Germany is ranked as the $7^{\text {th }}$ in the world's potato consumer list and Iran is the $10^{\text {th }}$ one. To test this idea, we need to conduct further studies on the low potato consumption rate countries such as African countries. The second possibility is that adults are allergic to potato in general. The answer to this question is open and that begs for further studies globally.

In contrast, cow milk, egg, soybean, and wheat are the most frequent observed allergen in food allergens group in children according to this study, which is consistent with some previous works but not with all. On the other hand, cow milk is the most widely consumed baby food in Iran. This suggests that the most frequent allergen in children might be related to the food behavior and eating style of the region of study.

Racial and ethnic differences may have an important role too. For instance, Benedict et al studied 2,084 children in
12 European countries (26). They have reported a variety of allergic patterns among those countries, and have concluded that the food allergens are generally more important in $\mathrm{AD}$ than inhaled allergens. According to Benedict's et al analysis, the most important inhaled allergens are house dust mite, cat, grass pollen, and Alternaria, in order from high to low, and our analysis also supports their conclusion. In this study, house dust mite, cat, horse, and dog also had a high frequency, that necessary training to contact prevention with animals and health measures to combat the mites is useful in these regions. Patch test methods that have been used to identify allergens have undoubtedly a decisive role; as of $\mathrm{Ow}$ czarek et al, they showed that the method used will make the difference in the prevalence of allergen (27). In this study we used immunoblotting, that is one of the newest methods for measurement of specific IgE. During a search of the Internet and of different similar sources of information, blot immunoassay method for the study of the spectrum of allergens was not found. As is evident, the distribution pattern of allergens in atopic dermatitis patients is highly variable, and the reason for this difference can be relevant to the differences between races, genetic, patch test methods, other different laboratory methods, vegetation and different eating habits, as this is proof of the necessity for detection of particular allergens in that particular region for the treatment of atopic dermatitis in the same area. In this study, frequency of allergens in patients was as follow: plants and fungi group $54.34 \%$, animal group $26.8 \%$ food groups $19.56 \%$. These results confirm the potential effect of inhaled allergens and plant allergens in patients with atopic dermatitis.

\section{Conclusions}

In this study, we used an immunoblotting technique, which is one of the most recent methods for the measurement of specific IgE, and the current study is the first on this topic which has used this technique. We believe that using this technique can revolutionize the identification of allergens, and significantly improve the currently presented treatment procedures to a higher level of quality and efficiency.

This is the first study in Azerbaijan which has focused on AD with the newest method that we have used. Our results would significantly improve the treatment procedure in Azerbaijan-Iran by focusing on the most frequent allergens in this region.

Based on the findings of this study, common allergens in East Azerbaijan, including cultivated rye, Timothy grass, house dust mite, Alternaria, cat, Cladosporium, horse, birch, potato, dog, were determined. With regard these findings, we can recommend to avoiding these allergens for atopic patients, and to use patient-specific allergy immunotherapy for treatments. 


\section{Acknowledgments}

This Study was supported by the grant no. 90-326 from Immunology Research Center, Faculty of medicine, Tabriz University of Medical Sciences. We would like to thank employees in the laboratory of Dr. Bonyadi.

\section{References}

1. Burns T, Breathnach S, Cox N, Griffiths C. Rook. Textbook of Dermatology. 4 Volume Set: Wiley;2010.

2. Bolognia J, Jorizzo JL, Rapini RP. Dermatology. Mosby/Elsevier; 2008.

3. Ou L-S, Huang J-L. Cellular aspects of atopic dermatitis. Clinical reviews in allergy \& immunology. 2007;33(3):191-8.

4. Borirakchanyavat K, Kurban AK. Atopic dermatitis. Clinics in dermatology. 2000;18(6):649-55. Epub 2001/02/15.

5. Kim T-E, Park S-W, Cho N-Y, Yong T, Nahm B, Lee S, et al. Quantitative measurement of serum allergen-specific $\operatorname{IgE}$ on protein chip. Experimental and molecular medicine. 2002;34(2):152-8.

6. Boguniewicz M, Schmid-Grendelmeier P, Leung DY. Atopic dermatitis. The Journal of allergy and clinical immunology. 2006;118(1):40-3. Epub 2006/07/04.

7. Sampson HA. The evaluation and management of food allergy in atopic dermatitis. Clinics in dermatology. 2003;21(3):183-92.

8. Warner J. Determinants of total and specific IgE in infants with atopic dermatitis. Pediatric Allergy and Immunology. 1997;8(4):177-84.

9. Perry TT, Matsui EC, Kay Conover-Walker M, Wood RA. The relationship of allergen-specific IgE levels and oral food challenge outcome. Journal of allergy and clinical immunology. 2004;114(1):144-9.

10. Hon KlE, Lam McA, Leung Tf, Wong Ky, Chow Cm, Fok Tf, et al. Are age-specific high serum IgE levels associated with worse symptomatology in children with atopic dermatitis? International journal of dermatology. 2007;46(12):1258-62.

11. Pádua I, Moreira A, Moreira P, Barros R. Food allergy: practical approach on education and accidental exposure prevention. European annals of allergy and clinical immunology. 2016;48(5):174.

12. Brand PL. Allergy diagnosis: pros and cons of different tests, indications and limitations. Breathe. 2007;3(4):345-9.

13. Westwood M, Ramaekers B, Lang S, Armstrong N, Noake C, de Kock S, Joore M, Severens J, Kleijnen J. ImmunoCAP ${ }^{\circledR}$ ISAC and Microtest for multiplex allergen testing in people with difficult to manage allergic disease: a systematic review and cost analysis. Health Technology Assessment (Winchester, England). 2016;20(67):1.

14. Wakamori T, Katoh N, Hirano S, Kishimoto S, Ozasa K. Atopic dermatitis, dry skin and serum IgE in children in a community in Japan. International archives of allergy and immunology. 2009;149(2):103-10.
15. Kulthanan K, Samutrapong P, Jiamton S, Tuchinda P. Adult-onset atopic dermatitis: a cross-sectional study of natural history and clinical manifestation. Asian Pacific Journal of Allergy and Immunology. 2010;25(4):207-14.

16. Kulthanan K, Boochangkool K, Tuchinda P, Chularojanamontri L. Clinical features of the extrinsic and intrinsic types of adult-onset atopic dermatitis. Asia Pacific Allergy. 2011;1(2):80.

17. Mgeladze N, Karseladze R, Zhorzholiani L, Karseladze E. Clinical-epidemiological characteristics of atopic dermatitis in children of junior school level in Tbilisi. Georgian medical news. 2009(167):68-71.

18. Sharma L. Diagnostic clinical features of atopic dermatitis. Indian Journal of Dermatology, Venereology, and Leprology. 2001;67(1):25.

19. De D, Kanwar A, Handa S. Comparative efficacy of Hanifin and Rajka's criteria and the UK working party's diagnostic criteria in diagnosis of atopic dermatitis in a hospital setting in North India. Journal of the European Academy of Dermatology and Venereology. 2006;20(7):853-9.

20. Böhme M, Svensson $\AA$, Kull I, Wahlgren C-F. Hanifin's and Rajka's minor criteria for atopic dermatitis: which do 2-year-olds exhibit? Journal of the American Academy of Dermatology. 2000;43(5):785-92.

21. Wahab M, Rahman M, Khondker L, Hawlader A, Ali A, Hafiz M, et al. Minor criteria for atopic dermatitis in children. Mymensingh medical journal: MMJ. 2011;20(3):419-24.

22. Ahmadi A, Sepehri S, Mosavinasab N, Torabi Z. Determination of Allergens Prevalence In allergic patients in Zanjan by Skin Prick Test. Journal of Zanjan University of Medical Sciences. 2008(54):25.

23. Worm M, Forschner K, Lee H-H, Roehr CC, Edenharter G, Niggemann B, et al. Frequency of atopic dermatitis and relevance of food allergy in adults in Germany. Acta dermato-venereologica. 2006;86(2).

24. Celakovska J, Vaneckova J, Ettlerova K, Ettler K, Bukac J. The role of atopy patch test in diagnosis of food allergy in atopic eczema/ dermatitis syndrom in patients over 14 years of age. Acta medica (Hradec Kralove) / Universitas Carolina, Facultas Medica Hradec Kralove. 2010;53(2):101-8. Epub 2010/08/03.

25. Hon KL, Chan IHS, Chow CM, Wang SS, Lam CWK, Ng PC, et al. Specific IgE of common foods in Chinese children with eczema. Pediatric Allergy and Immunology. 2011;22(1-Part-I):50-3.

26. De Benedictis F, Franceschini F, Hill D, Naspitz C, Simons F, Wahn U, et al. The allergic sensitization in infants with atopic eczema from different countries. Allergy. 2009;64(2):295-303.

27. Owczarek W, Paluchowska E, Targowski T. [The relation of atopy patch tests with immunoglobulin E. Atopy patch tests with aeroallergens results dependence with the concentration of specific class $\mathrm{E}$ antibodies assay level assessment]. Polski merkuriusz lekarski: organ Polskiego Towarzystwa Lekarskiego. 2009;26(151):35-9. 